\title{
LINEAR OPERATORS ON QUASI-CONTINUOUS FUNCTIONS
}

\author{
BY \\ RALPH E. LANE
}

1. Introduction. In this paper we study a class of linear transformations for each of which the transform of a function is a function. This special class of transformations has such applications as the smoothing of experimental data, the prediction of outputs of a physical system for various inputs, and the estimation of the velocities and accelerations of an object from observations of its positions at various times.

By the statement that $f$ is a function, we imply that if $t$ is a real number, then $f(t)$ is a number. By the statement that $f$ is quasi-continuous $\left({ }^{1}\right)$, we mean that $f$ is a function such that if $t$ is a real number then the limits $f(t-)$ and $f(t+)$ exist. Some lemmas on quasi-continuous functions appear in $\S 2$.

Definition 1.1. The statement that $T$ is a $Q$ operator over the interval $[a, b]$ means that $T$ is a transformation such that

(i) if $y$ is quasi-continuous, then $T y$ is a function; if $g=T y$ and $s$ is a real number, then we denote the number $g(s)$ by $T y(s)$,

(ii) if $y_{1}$ is quasi-continuous and $y_{2}$ is quasi-continuous, then $T\left(y_{1}+y_{2}\right)$ $=T y_{1}+T y_{2}$,

(iii) if $y$ is quasi-continuous and $k$ is a number, then $T(k y)=k(T y)$,

(iv) if $y$ is quasi-continuous, $c$ is a real number, and $z(t)=y(t+c)$ for each real number $t$, then $T z(s)=T y(s+c)$ for each real number $s$, and

(v) if $s$ is a real number, then there is a positive number $B_{s}$ such that if $y$ is quasi-continuous and $M>|y(s-t)|$ for each number $t$ in $[a, b]$, then $|T y(s)| \leqq M B_{s}$; by the norm, $|T(s)|$, of $T$ at $s$ we mean the greatest lower bound of the set of all such numbers $B_{s}$.

It will be observed that if $T$ is a $Q$ operator over the interval $[a, b]$ and $T_{0} y=T y(0)$ for each quasi-continuous function $y$, then $T_{0}$ is a bounded linear transformation from the set of all quasi-continuous functions to the set of all numbers (i.e., $T_{0}$ is a bounded linear functional operation as defined in [2] and [3]). We give the following example of a $Q$ operator.

EXAMPLE 1.1. Suppose that if $y$ is quasi-continuous and $s$ is a real number, then

$$
\begin{aligned}
T y(s)=[-y(s-2)-3 y(s-1)+76 y(s) & +76 y(s+1) \\
& -3 y(s+2)-y(s+3)] / 144 .
\end{aligned}
$$

Presented to the Society April 21, 1956; received by the editors August 16, 1956.

(1) Except for the use of the word "quasi-continuous," we use the terminology and notation of [1]. In particular, "integral" is defined as in [1]. 
It follows that $T$ is a $Q$ operator over the interval $[-3,2]$ and that $|T(s)|$ $=10 / 9$ for each real number $s$. Moreover, if $y$ is a polynomial of degree 3 or less, then $T y(s)=\int_{s}^{s+1} y(t) d t$. This operator is designed for use with experimental data, for which the values used for $y$ in the formula may include errors of observation. In effect, the operator smooths the raw data, interpolates, and gives the integral of the smoothed and interpolated data; it is derived from Jenkins' modified osculatory interpolation formula [4].

In $\S 3$ we show that if $T$ is a $Q$ operator over the interval $[a, b]$, then $T y$ is quasi-continuous, of bounded variation, or continuous, according as $y$ is quasi-continuous, of bounded variation, or continuous. In $\$ 4$ we show that if $T$ is a $Q$ operator and $y$ is a quasi-continuous function then $T y$ is the sum of two integrals.

We adopt the following notation. If $y$ is quasi-continuous, then $y_{L}$ and $y_{R}$ denote the functions such that $y_{L}(t)=y(t-)$ and $y_{R}(t)=y(t+)$ for each real number $t$.

Definition 1.2. The statement that $T$ is a $Q_{1}$ operator over the interval $[a, b]$ means that $T$ is a $Q$ operator over $[a, b]$ such that if $y$ is quasi-continuous then $T y(s+)-T y(s-)=2\left[T y_{R}(s)-T y_{L}(s)\right]$ for each real number $s$; i.e., if $T y=x, T\left(y_{L}\right)=u$ and $T\left(y_{R}\right)=v$, then $x(s+)-x(s-)=2[v(s)-u(s)]$ for each real number $s$.

In $\S 5$ we show that a $Q_{1}$ operator $T$ is a $Q$ operator such that if $y$ is a quasi-continuous function then $T y$ is an integral. In $\S 6$ we find conditions sufficient to assure that a $Q_{1}$ operator $T$ has various properties which may be desirable in applications. For example, we find a condition sufficient to assure that if $y$ is quasi-continuous then $T y$ has a derivative, and we exhibit a $Q_{1}$ operator $T^{\prime}$ such that $T^{\prime} y$ is the derivative of $T y$. In $\S 7$ we give a family of $Q_{1}$ operators, one of which is a limit of the "most powerful" smoothing operators given in [4].

2. Lemmas concerning quasi-continuous functions. The following results will be used later in this paper.

Lemma 2.1. For the function $f$ to be quasi-continuous, it is necessary and sufficient that if $[a, b]$ is an interval and $\epsilon>0$ then there is a step-function $s$ such that $|f(t)-s(t)|<\epsilon$ for each number $t$ in $[a, b]$.

For a proof, see Lemma 4.1b of [1]; see [5] also.

Lemma 2.2. For the function $f$ to be quasi-continuous it is necessary and sufficient that if $[a, b]$ is an interval and $\epsilon>0$ then there is a subdivision $t_{0}$, $t_{1}, \cdots, t_{n}$ of $[a, b]$ such that if $p$ and $q$ are in one of the segments $\left(t_{i}, t_{i+1}\right)$ then $|f(p)-f(q)|<\epsilon$.

Proof is omitted.

Lemma 2.3. If $f_{1}, f_{2}, f_{3}, \cdots$ is a sequence of quasi-continuous functions which converges uniformly to a function $f$, then $f$ is quasi-continuous. Moreover, 
if $f_{n}(t+)=f_{n}(t)$ for each positive integer $n$ and each real number $t$, then $f_{R}=f$; likewise, if $f_{n}(t-)=f_{n}(t)$ for each positive integer $n$ and each real number $t$, then $f_{L}=f$.

Proof is omitted.

Lemma 2.4. If $f_{1}, f_{2}, \cdots$, is a sequence of functions which converges uniformly to a function $f$, and $[a, b]$ is an interval, and $V>0$, and $V_{a}^{b}\left(f_{n}\right) \leqq V$ for each positive integer $n$, then $V_{a}^{b}(f) \leqq V$.

For a proof, see Lemma $4.2 \mathrm{a}$ of [1].

LEMma 2.5. If $f$ is a quasi-continuous function, then there are a quasicontinuous function $g$ and a quasi-continuous function $h$ such that

(i) $g_{L}=g$ and $h_{R}=h$,

(ii) $g+h=f$, and

(iii) if $[a, b]$ is an interval and $|f(t)| \leqq M<M_{1}$ for each number $t$ in $[a, b]$, then $|g(t)| \leqq 1.5 M_{1}$ and $|h(t)| \leqq 1.5 M_{1}$ for each number $t$ in $[a, b]$.

Proof. We introduce the following notation. Suppose that $[a, b]$ is an interval, $z$ is quasi-continuous, and $D$ is a subdivision $t_{0}, t_{1}, \cdots, t_{m}$ of $[a, b]$. Then $L_{D} z$ and $R_{D} z$ denote the pair of functions such that $2 L_{D} z(a)=z(a)$, $2 R_{D} z(b)=z(b)$, and for $i=0,1, \cdots, m-1$,

$$
2\left(t_{i+1}-t_{i}\right) L_{D} z(t)=\left(t_{i+1}-t\right)\left[2 z\left(t_{i}+\right)-z\left(t_{i}\right)\right]+\left(t-t_{i}\right) z\left(t_{i+1}\right) \text { if } t_{i}<t \leqq t_{i+1},
$$

and

$$
2\left(t_{i+1}-t_{i}\right) R_{D} z(t)=\left(t_{i+1}-t\right) z\left(t_{i}\right)+\left(t-t_{i}\right)\left[2 z\left(t_{i+1}-\right)-z\left(t_{i+1}\right)\right] \text { if } t_{i} \leqq t<t_{i+1} \text {. }
$$

We make the following three observations. First, if $|z(t)| \leqq M$ for each number $t$ in $[a, b]$, then $\left|L_{D} z(t)\right| \leqq 1.5 M$ and $\left|R_{D} z(t)\right| \leqq 1.5 M$ for $a \leqq t \leqq b$. Second, if $\epsilon>0$ and it is true that $|z(p)-z(q)| \leqq \epsilon$ if $p$ and $q$ are in one of the segments $\left(t_{i}, t_{i+1}\right)$, then $\left|z(t)-L_{D} z(t)-R_{D} z(t)\right| \leqq \epsilon$ for $a \leqq t \leqq b$. Finally, $L_{D} z(t-)=L_{D} z(t)$ if $a<t \leqq b$, and $R_{D} z(t+)=R_{D} z(t)$ if $a \leqq t<b$.

Now suppose that $[a, b]$ is an interval and $|f(t)| \leqq M<M_{1}$ if $a \leqq t \leqq b$. Let $\epsilon$ denote a positive number less than $M_{1}-M$. We now construct a sequence $f_{1}, f_{2}, f_{3}, \cdots$ of functions and a sequence $D_{1}, D_{2}, D_{3}, \cdots$ of subdivisions of $[a, b]$ in the following manner. Let $f_{1}$ denote the function $f$, and let $D_{1}$ denote a subdivision $t_{0}, t_{1}, \cdots, t_{m}$ of $[a, b]$ such that if $p$ and $q$ are in one of the segments $\left(t_{i}, t_{i+1}\right)$ then $\left|f_{1}(p)-f_{1}(q)\right|<\epsilon / 2$. For each positive integer $n$, let $f_{n+1}=f_{n}-L_{D_{n}} f_{n}-R_{D_{n}} f_{n}$, where $D_{n}$ denotes a subdivision $t_{0}, t_{1}, \cdots, t_{m}$ of $[a, b]$ such that if $p$ and $q$ are in one of the segments $\left(t_{i}, t_{i+1}\right)$ then $\mid f_{n}(p)$ $-f_{n}(q) \mid \leqq \epsilon / 2^{n}$. Suppose that $a \leqq t \leqq b$. Then $\left|f_{1}(t)\right| \leqq M$, and therefore $\left|L_{D_{1}} f_{1}(t)\right| \leqq 1.5 M$ and $\left|R_{D_{1}} f_{1}(t)\right| \leqq 1.5 M$; moreover, $\left|f_{2}(t)\right|=\mid f_{1}(t)-L_{D_{1}} f_{1}(t)$ $-R_{D_{1}} f_{1}(t) \mid \leqq \epsilon / 2$. By induction, if $n$ is an integer greater than 1 , and $a \leqq t \leqq b$, then $\left|L_{D_{n}} f_{n}(t)\right| \leqq 1.5 \epsilon / 2^{n-1},\left|R_{D_{n}} f_{n}(t)\right| \leqq 1.5 \epsilon / 2^{n-1}$, and 


$$
\begin{aligned}
\mid f_{1}(t)-\sum_{p=1}^{n} L_{D_{p}} f_{p}(t) & -\sum_{p=1}^{n} R_{D_{p}} f_{p}(t) \mid \\
& =\left|f_{n+1}(t)\right|=\left|f_{n}(t)-L_{D_{n}} f_{n}(t)-R_{D_{n}} f_{n}(t)\right| \leqq \epsilon / 2^{n} .
\end{aligned}
$$

But $\sum_{p=1}^{\infty} L_{D_{p}} f_{p}$ converges uniformly in $[a, b]$ to a function $g$; and if $a \leqq t \leqq b$, then $|g(t)| \leqq \sum_{p=1}^{\infty}\left|L_{D_{p}} f_{p}(t)\right| \leqq 1.5(M+\epsilon)<1.5 M_{1}$. Similarly, $\sum_{p=1}^{\infty} R_{D_{p}} f_{p}$ converges uniformly in $[a, b]$ to a function $h$, and if $a \leqq t \leqq b$ then $|h(t)|$ $<1.5 M_{1}$. Moreover, if $a \leqq t \leqq b$ then $g(t)+h(t)=f(t)$. From Lemma 2.3, it follows that if $a<t \leqq b$ then $h(t-)$ exists and $g(t-)=g(t)$, and that if $a \leqq t<b$ then $g(t+)$ exists and $h(t+)=h(t)$. This completes the proof.

Lemma 2.6. Suppose that $\left[t_{0}, t_{1}\right]$ is an interval, $\epsilon>0$, and $f$ is a function such that

(i) if $t_{0} \leqq t \leqq t_{1}$, then the derivative $f^{\prime}(t)$ exists, and

(ii) if $s_{1}$ and $s_{2}$ are in $\left[t_{0}, t_{1}\right]$, then $\left|f^{\prime}\left(s_{1}\right)-f^{\prime}\left(s_{2}\right)\right|<\epsilon$. Then

$$
\left|\left(f\left(t_{1}\right)-f\left(t_{0}\right)\right) /\left(t_{1}-t_{0}\right)-f^{\prime}(t)\right|<\epsilon(2)^{1 / 2}
$$

for each number $t$ in $\left[t_{0}, t_{1}\right]$.

Proof is omitted, since this lemma can be obtained by applying the theorem of the mean to the real part and the imaginary part of $f$.

LEMMA 2.7. If $f$ has a quasi-continuous derivative $f^{\prime}$, then $f^{\prime}$ is continuous.

Proof is omitted, since the lemma follows readily from well-known results and can be derived from Lemma 2.6.

Lemma 2.8. Suppose that $[a, b]$ is an interval, $y$ is a function which is bounded in $[a, b]$, and $x$ is a function whose derivative, $x^{\prime}$, is continuous in $[a, b]$. If $\int_{a}^{b} y(t) d x(t)=I$ or $\int_{a}^{b} y(t) x^{\prime}(t) d t=I$, then $\int_{a}^{b} y(t) d x(t)=\int_{a}^{b} y(t) x^{\prime}(t) d t$.

Proof is omitted, since this lemma follows with little difficulty from Lemma 2.6.

Lemma 2.9. Suppose that $f$ is a function whose derivative, $f^{\prime}$, is of bounded variation in the interval $[a, b], h$ is a real number other than zero, and $[c, d]$ is an interval such that if $t$ is in $[c, d]$ then $t$ and $t+h$ are in $[a, b]$. If $g(t)$ $=[f(t+h)-f(t)] / h$ for each number $t$ in $[c, d]$, then $g$ is of bounded variation in $[c, d]$, and $V_{c}^{d}(g) \leqq V_{a}^{b}\left(f^{\prime}\right)$.

Proof. Suppose that $t_{0}, t_{1}, \cdots, t_{n}$ is a subdivision of $[c, d]$, and let $S$ denote the sum $\sum_{i=0}^{n-1}\left|g\left(t_{i+1}\right)-g\left(t_{i}\right)\right|$. Now

$$
\begin{aligned}
h\left[g\left(t_{i+1}\right)-g\left(t_{i}\right)\right] & =\int_{0}^{h} 1 d f\left(t_{i+1}+t\right)-\int_{0}^{h} 1 d f\left(t_{i}+t\right) \\
& =\int_{0}^{h}\left[f^{\prime}\left(t_{i+1}+t\right)-f^{\prime}\left(t_{i}+t\right)\right] d t .
\end{aligned}
$$


It follows that if $h>0$, then

$$
h\left|g\left(t_{i+1}\right)-g\left(t_{i}\right)\right| \leqq \int_{0}^{h}\left|f^{\prime}\left(t_{i+1}+t\right)-f^{\prime}\left(t_{i}+t\right)\right| d t
$$

and

$$
h S \leqq \int_{0}^{h} \sum_{i=0}^{n-1}\left|f^{\prime}\left(t_{i+1}+t\right)-f^{\prime}\left(t_{i}+t\right)\right| d t \leqq \int_{0}^{h} V_{a}^{b}\left(f^{\prime}\right) d t=h V_{a}^{b}\left(f^{\prime}\right),
$$

so that $S \leqq V_{a}^{b}\left(f^{\prime}\right)$. By a similar argument, if $h<0$, then $S \leqq V_{a}^{b}\left(f^{\prime}\right)$. Hence $g$ is of bounded variation in $[c, d]$, and $V_{c}^{d}(g) \leqq V_{a}^{b}\left(f^{\prime}\right)$. This completes the proof.

3. Some properties of $Q$ operators. In this section we suppose that there are given an interval $[a, b]$ and a $Q$ operator $T$ over $[a, b]$.

Lemma 3.1a. Suppose that $s$ is a real number and $y$ and $z$ are quasi-continuous functions such that $y(s-t)=z(s-t)$ if $a \leqq t \leqq b$. Then $T y(s)=T z(s)$.

Proof. Let $w=y-z$. By (ii) and (iii) of Definition 1.1, $T y(s)-T z(s)$ $=T w(s)$. But $w(s-t)=0$ if $a \leqq t \leqq b$; and by (v) of Definition 1.1, if $\epsilon>0$ then $|T w(s)| \leqq \epsilon|T(s)|$; hence $T w(s)=0$, or $T y(s)=T z(s)$. This completes the proof.

THEOREM 3.1. If $s$ is a real number, then $|T(s)|=|T(0)|$.

Proof. Suppose that $s$ is a real number. Now if $y$ is quasi-continuous and $z(t)=y(t-s)$ for each real number $t$, then $z(s-t)=y(-t)$ for each number $t$ in $[a, b]$; by Lemma 3.1a and (iv) of Definition 1.1, $T z(s)=T y(0)$. It follows from (v) of Definition 1.1 that $|T(s)|=|T(0)|$. This completes the proof.

REMARK. In view of Theorem 3.1, we shall hereafter refer to the norm of $T$ as $|T|$; i.e., if $s$ is a real number, then $|T|=|T(s)|$.

THEOREM 3.2. If $a<c<b$, then there are a $Q$ operator $T_{1}$ over $[a, c]$ and $a Q$ operator $T_{2}$ over $[c, b]$ such that

(i) if $y$ is quasi-continuous then $T y=T_{1} y+T_{2} y$, and

(ii) $\left|T_{1}\right|+\left|T_{2}\right|=|T|$.

Proof. If $y$ is quasi-continuous and $s$ is a real number, then we define numbers $T_{1} y(s)$ and $T_{2} y(s)$ in the following manner. Let $u(s-t)=0$ if $t>c$ and $u(s-t)=y(s-t)$ if $t \leqq c$ and let $T_{1} y(s)=T u(s)$. Let $v(s-t)=y(s-t)$ if $t>c$ and $v(s-t)=0$ if $t \leqq c$; and let $T_{2} y(s)=T v(s)$. By Definition 1.1, $T_{1}$ is a $Q$ operator over $[a, c]$, and $T_{2}$ is a $Q$ operator over $[c, b]$; moreover, if $y$ is quasi-continuous, then $T_{1} y+T_{2} y=T y$.

We now show that $\left|T_{1}\right|+\left|T_{2}\right| \geqq|T|$. Suppose that $\epsilon>0, s$ is a real number and $y$ is a quasi-continuous function such that $|T y(s)|>|T|-\epsilon$ and $|y(s-t)| \leqq 1$ for each number $t$ in $[a, b]$. Now $\left|T_{1}\right|+\left|T_{2}\right| \geqq\left|T_{1} y(s)\right|$ $+\left|T_{2} y(s)\right| \geqq\left|T_{1} y(s)+T_{2} y(s)\right|=|T y(s)|>|T|-\epsilon$. Hence $\left|T_{1}\right|=T_{1}+\left|T_{2}\right|$ $\geqq|T|$.

Finally we show that $\left|T_{1}\right|+\left|T_{2}\right| \leqq|T|$. Suppose that $\epsilon>0, s$ is a real 
number, and $z$ is a quasi-continuous function such that $T_{1} z(s)=\left|T_{1} z(s)\right|$ $>\left|T_{1}\right|-\epsilon, T_{2} z(s)=\left|T_{2} z(s)\right|>\left|T_{2}\right|-\epsilon$, and $|z(s-t)| \leqq 1$ if $t$ is in $[a, b]$. Now $|T| \geqq|T z(s)|=\left|T_{1} z(s)+T_{2} z(s)\right|=T_{1} z(s)+T_{2} z(s)>\left|T_{1}\right|+\left|T_{2}\right|-2 \epsilon$. Hence $|T| \geqq\left|T_{1}\right|+\left|T_{2}\right|$. This completes the proof.

Lemma 3.3a. There is a number sequence $c_{1}, c_{2}, c_{3}$ such that if $s$ is a real number and $y$ is a step-function such that $y(s-p)=y(s-q)$ for each pair $p, q$ of numbers between $a$ and $b$, then $T y(s)=c_{1} y(s-b)+c_{2} y(s-b+)+c_{3} y(s-a)$; moreover, $|T| \geqq\left|c_{1}\right|+\left|c_{2}\right|+\left|c_{3}\right|$.

Proof. Let functions $u, v, w$ be defined as follows:

$$
\begin{aligned}
& u(-t)=1 \text { if } t \geqq b \text { and } u(-t)=0 \text { if } t<b ; \\
& v(-t)=1 \text { if } a<t<b \text { and } v(-t)=0 \text { if } t \geqq b \text { or } t \leqq a ; \text { and } \\
& w(-t)=1 \text { if } t \leqq a \text { and } w(-t)=0 \text { if } t>a .
\end{aligned}
$$

Let $c_{1}=T u(0), c_{2}=T v(0)$, and $c_{3}=T w(0)$. Now if $s$ is a real number, $a \leqq t \leqq b$, and $y$ is a step-function such that $y(s-p)=y(s-q)$ for each pair $p, q$ of numbers between $a$ and $b$, then $y(s-t)=y(s-b) u(-t)+y(s-b+) v(-t)+y(s-a)$ $w(-t)$, and therefore $T y(s)=c_{1} y(s-b)+c_{2} y(s-b+)+c_{3} y(s-a)$.

Now let $d_{1}, d_{2}, d_{3}$ denote a number sequence such that $\left|d_{1}\right|=\left|d_{2}\right|=\left|d_{3}\right|=1$ and $c_{1} d_{1} \geqq 0, c_{2} d_{2} \geqq 0$, and $c_{3} d_{3} \geqq 0$. For each real number $t$, let $z(t)=d_{1} u(t)$ $+d_{2} v(t)+d_{3} w(t)$. Now if $a \leqq t \leqq b$, then $|z(-t)|=1$, so that $|T z(0)| \leqq|T|$; but $T z(0)=c_{1} d_{1}+c_{2} d_{2}+c_{3} d_{3}=\left|c_{1}\right|+\left|c_{2}\right|+\left|c_{3}\right|$; so $|T| \geqq\left|c_{1}\right|+\left|c_{2}\right|+\left|c_{3}\right|$. This completes the proof.

Lemma 3.3b. Suppose that $t_{0}, t_{1}, \cdots, t_{2 n}$ is a subdivision of $[a, b]$. There is a number sequence $c_{0}, c_{1}, \cdots, c_{2 n}$ such that if $s$ is a real number and $y$ is a stepfunction such that $y(s-p)=y(s-q)$ for each pair $p, q$ of numbers between $t_{22}$ and $t_{2 i+2}, i=0,1, \cdots, n-1$, then $T y(s)=\sum_{i=0}^{2 n} c_{i} y\left(s-t_{i}\right) ;$ moreover, $|T|$ $\geqq \sum_{i=0}^{2 n}\left|c_{i}\right|$.

Proof is omitted, since this lemma follows from Theorem 3.2 and Lemma 3.3a.

Lemma 3.3c. If $y$ is a step-function and $[c, d]$ is an interval, then Ty is of bounded variation in $[c, d]$, and $V_{c}^{d}(T y) \leqq|T| \cdot V_{c-b}^{d-a}(y)$.

Proof. Let $s_{0}, s_{1}, \cdots, s_{m}$ denote a subdivision of $[c, d]$. Let $t_{0}, t_{1}, \cdots, t_{2 n}$ denote a subdivision of $[a, b]$ such that if $j$ is one of the integers $0,1, \cdots, m$, and $i$ is one of the integers $0,1, \cdots, n-1$, and $p$ and $q$ are numbers between $t_{2 i}$ and $t_{2 i+2}$, then $y\left(s_{j}-p\right)=y\left(s_{j}-q\right)$. By Lemma $3.3 \mathrm{~b}$, there is a number sequence $c_{0}, c_{1}, \cdots, c_{2 n}$ such that $T y\left(s_{j}\right)=\sum_{i=0}^{2 n} c_{i} y\left(s_{j}-t_{i}\right)$ for $j=0,1, \cdots, m$, and $|T| \geqq \sum_{i=0}^{2 n}\left|c_{i}\right|$. Now if $j$ is one of the integers $0,1, \cdots, m-1$, then $T y\left(s_{j}+1\right)-T y\left(s_{j}\right)=\sum_{t=0}^{2 n} c_{i}\left[y\left(s_{j+1}-t_{i}\right)-y\left(s_{j}-t_{i}\right)\right]$. Hence 


$$
\begin{aligned}
\sum_{j=0}^{m-1}\left|T y\left(s_{j+1}\right)-T y\left(s_{j}\right)\right| & =\sum_{j=0}^{m-1}\left|\sum_{i=0}^{2 n} c_{i}\left[y\left(s_{j+1}-t_{i}\right)-y\left(s_{j}-t_{i}\right)\right]\right| \\
& \leqq \sum_{j=0}^{m-1} \sum_{i=0}^{2 n}\left|c_{i}\right| \cdot\left|y\left(s_{j+1}-t_{i}\right)-y\left(s_{j}-t_{i}\right)\right| \\
& \leqq \sum_{i=0}^{2 n}\left|c_{i}\right| \cdot V_{c-t_{i}}^{d-t_{i}}(y) \\
& \leqq|T| \cdot V_{c-b}^{d-a}(y) .
\end{aligned}
$$

The lemma now follows at once.

THEOREM 3.3. If $y$ is quasi-continuous, then Ty is quasi-continuous; if $y$ is of bounded variation, then Ty is of bounded variation; if $y$ is continuous, then Ty is continuous.

Proof. Suppose first that $y$ is quasi-continuous. Let $y_{1}, y_{2}, y_{3}, \cdots$ denote a sequence of step-functions which converges uniformly to $y$. By (v) of Definition $1.1, T y_{1}, T y_{2}, T y_{3}, \cdots$ converges uniformly to $T y$. Now if $[c, d]$ is an interval and $n$ is a positive integer, then by Lemma 3.3c, $T y_{n}$ is of bounded variation in $[c, d]$. Hence each of the functions $T y_{i}$ is quasi-continuous; by Lemma 2.3, $T y$ is quasi-continuous.

Suppose now that $y$ is of bounded variation, and that $[c, d]$ is an interval, and $V \geqq V_{c-b}^{d-a}(y)$. Let $y_{1}, y_{2}, y_{3}, \cdots$ denote a sequence of step-functions converging uniformly to $y$ such that $V_{c-b}^{d-a}\left(y_{n}\right) \leqq V, n=1,2,3, \cdots$. Then $T y_{1}, T y_{2}, T y_{3}, \cdots$ is a sequence of functions converging to $T y$ uniformly, and $V_{c}^{d}\left(T y_{n}\right) \leqq|T| V$ for $n=1,2,3, \cdots$. By Lemma $2.4, V_{c}^{d}(T y) \leqq|T| V$; hence $T y$ is of bounded variation.

Suppose, finally, that $y$ is continuous and that $[c, d]$ is an interval. Let $\epsilon$ denote a positive number, and let $\delta$ denote a positive number such that if $c-b \leqq p<q \leqq d-a$ and $q-p<\delta$, then $|y(p)-y(q)|<\epsilon$. Now suppose that $c \leqq s_{1}<s_{2} \leqq d$ and $s_{2}-s_{1}<\delta$. If $a \leqq t \leqq b$, then $\left|y\left(s_{1}-t\right)-y\left(s_{2}-t\right)\right|<\epsilon$; so $\left|T y\left(s_{1}\right)-T y\left(s_{2}\right)\right|<\epsilon|T|$. Hence $T y$ is continuous. This completes the proof.

Corollary 3.3a. If $T_{1}$ is a $Q$ operator over the interval $\left[a_{1}, b_{1}\right]$ and $T_{2}$ is a $Q$ operator over the interval $\left[a_{2}, b_{2}\right]$, and $T_{3} y=T_{1}\left(T_{2} y\right)$ for each quasi-continuous function $y$, then $T_{3}$ is a $Q$ operator over the interval $\left[a_{1}+a_{2}, b_{1}+b_{2}\right]$, and $\left|T_{3}\right|$ $\leqq\left|T_{1}\right| \cdot\left|T_{2}\right|$.

Proof. By Theorem 3.3, if $y$ is quasi-continuous, then so is $T_{2} y$; therefore $T_{3} y$ is a function. It can readily be verified that $T_{3}$ has the properties listed as (ii), (iii), and (iv) in Definition 1.1. Let $s$ denote a real number, suppose that $y$ is quasi-continuous, and that $M \geqq|y(s-t)|$ if $a_{1}+a_{2} \leqq t \leqq b_{1}+b_{2}$. Now if $a_{1} \leqq s_{1} \leqq b_{1}$ and $a_{2} \leqq t \leqq b_{2}$, then $a_{1}+a_{2} \leqq s_{1}+t \leqq b_{1}+b_{2}$; hence $\left|y\left(s-s_{1}-t\right)\right|$ $\leqq M$ if $a_{1} \leqq s_{1} \leqq b_{1}$ and $a_{2} \leqq t \leqq b_{2}$, so that $\left|T_{2} y\left(s-s_{1}\right)\right| \leqq\left|T_{2}\right| M$ if $a_{1} \leqq s_{1} \leqq b_{1}$, and therefore $\left|T_{3} y(s)\right| \leqq\left|T_{1}\right| \cdot\left|T_{2}\right| M$. Hence $T_{3}$ is a $Q$ operator over $\left[a_{1}+a_{2}\right.$, 
$\left.b_{1}+b_{2}\right]$, and $\left|T_{3}\right| \leqq\left|T_{1}\right| \cdot\left|T_{2}\right|$. This completes the proof.

Example 3.1. We give an example of Corollary 3.3a for which $\left|T_{3}\right|$ $<\left|T_{1}\right| \cdot\left|T_{2}\right|$. Suppose that if $y$ is quasi-continuous and $s$ is a real number, then $T_{1} y(s)=\left[y(s-)+y(s)^{\circ}\right] / 2$ and $T_{2} y(s)=[y(s)-y(s+)] / 2$. Then $T_{3} y(s)$ $=[y(s)-y(s+)] / 4$. Hence $\left|T_{1}\right|=1,\left|T_{2}\right|=1$, and $\left|T_{3}\right|=1 / 2$.

4. Integral representation of $Q$ operators. In this section we suppose that there are given an interval $[a, b]$ and a $Q$ operator over $[a, b]$. We introduce the following notation:

$$
\begin{aligned}
& J(t)=0 \text { if } t<0 \text { and } J(t)=1 \text { if } t \geqq 0, \\
& L(s)=T J_{L}(s) \text { and } R(s)=T J_{R}(s)=T J(s) \text { for each real number } s, \\
& x_{1}(t)=2 L(t)-L(t-) \text { and } x_{2}(t)=2 R(t)-R(t+) \text { for each real number } t .
\end{aligned}
$$

We note in passing that $L(t)=\left[x_{1}(t-)+x_{1}(t)\right] / 2$ and $R(t)=\left[x_{2}(t)+x_{2}(t+)\right] / 2$.

LEMMA 4.1a. $R(s)=L(s)=L(a)=0$ if $s<a$; and $R(s)=L(s)=R(b)$ if $s>b$. Moreover, $R\left(s_{-}\right)=L(s-)$ and $R(s+)=L(s+)$ for each real number $s$.

Proof. If $s<a$ and $a \leqq t \leqq b$, then $s-t<0$, so that $J_{L}(s-t)=J_{R}(s-t)$ $=J_{L}(a-t)=0$, and therefore $L(s)=R(s)=L(a)=0$. If $s>b$ and $a \leqq t \leqq b$, then $s-t>0$, so that $J_{L}(s-t)=J_{R}(s-t)=J_{R}(b-t)=1$, and therefore $L(s)=R(s)$ $=R(b)$.

Suppose now that $s$ is a real number. Let $s_{1}, s_{2}, s_{3}, \cdots$ denote a decreasing sequence which converges to $s$. Let $a_{1}, a_{2}, a_{3}, \cdots$ denote a number sequence such that if $p$ is a positive integer then $\left|a_{p}\right|=1$ and $a_{p}\left[R\left(s_{p}\right)-L\left(s_{p}\right)\right] \geqq 0$. For each positive integer $n$, let $f_{n}$ denote the step-function such that if $t$ is a real number then $f_{n}(t)=\sum_{p=1}^{n} a_{p}\left[J_{R}\left(s_{p}+t\right)-J_{L}\left(s_{p}+t\right)\right]$. Then $T f_{n}(0)$ $=\sum_{p=1}^{n}\left|R\left(s_{p}\right)-L\left(s_{p}\right)\right|$. But if $a \leqq t \leqq b$, then $\left|f_{n}(-t)\right| \leqq 1$, whence $\left|T f_{n}(0)\right|$ $\leqq|T|$. Hence $\sum_{p=1}^{n}\left|R\left(s_{p}\right)-L\left(s_{p}\right)\right|$ converges, and therefore $\left|R\left(s_{p}\right)-L\left(s_{p}\right)\right|$ $\rightarrow 0$ as $p \rightarrow+\infty$. It follows that $R(s+)=L(s+)$. By a similar argument, if $s$ is a real number, then $R(s-)=L(s-)$. This completes the proof.

Lemma $4.1 \mathrm{~b}$. If $s$ is a real number, then

$$
L(s)=\int_{a}^{b+} J_{L}(s-t) d x_{1}(t)
$$

and

$$
R(s)=\int_{a-}^{b} J_{R}(s-t) d x_{2}(t) .
$$

Proof. By Lemma 4.1a, $x_{1}(t)=x_{2}(t)=x_{1}(a)=0$ if $t<a$; and $x_{1}(t)=x_{2}(t)$ $=x_{2}(b)$ if $t>b$. Moreover, by Theorem 3.3, $R$ and $L$ are of bounded variation, and therefore $x_{1}$ and $x_{2}$ are of bounded variation. Suppose that $s$ is a real number and $p$ is a positive number such that $p>s-b$ and $p>a-s$. Then $\int_{a}^{b+} J_{L}(s-t) d x_{1}(t)=\int_{a-p}^{b+p} J_{L}(s-t) d x_{1}(t)$. By the integration by parts formula, 


$$
\begin{aligned}
\int_{a-p}^{b+p} J_{L}(s-t) d x_{1}(t)= & J_{L}(s-b-p) x_{1}(b+p)-J_{L}(s-a+p) x_{1}(a-p) \\
& -\int_{a-p}^{b+p} x_{1}(t) d J_{L}(s-t),
\end{aligned}
$$

or

$$
\int_{a}^{b+} J_{L}(s-t) d x_{1}(t)=\left[x_{1}(s-)+x_{1}(s)\right] / 2=L(s) .
$$

By a similar argument,

$$
\int_{a-}^{b} J_{R}(s-t) d x_{2}(t)=\left[x_{2}(s)+x_{2}(s+)\right] / 2=R(s) .
$$

THEOREM 4.1. Suppose that $y$ is quasi-continuous and $s$ is a real number. Let $g$ and $h$ denote quasi-continuous functions such that $g_{L}=g, h_{R}=h$, and $g+h$ $=y$. Then

$$
T y(s)=\int_{a}^{b+} g(s-t) d x_{1}(t)+\int_{a-}^{b} h(s-t) d x_{2}(t) .
$$

Proof. If $g$ is a step-function, it follows from Lemma $4.1 \mathrm{~b}$ that $T g(s)$ $=\int_{a}^{b+} g(s-t) d x_{1}(t)$; if $g$ is not a step-function, it follows from Lemmas 2.3 and $4.1 \mathrm{~b}$ of the present paper and Lemma 4.1a of [1] that $T g(s)=\int_{a}^{b+} g(s-t) d x_{1}(t)$. Similarly, $T h(s)=\int_{a-}^{b} h(s-t) d x_{2}(t)$. The theorem now follows from (ii) of Definition 1.1.

REMARK 4.1. Upon comparing Theorem 4.1 with (v) of Definition 1.1, one might suppose that $x_{1}(b+)=x_{1}(b)$ and $x_{2}(a-)=x_{2}(a)$. To show that this need not be so, we give the following example. For each quasi-continuous function $y$ and real number $s$, let $T y(s)=[y(s)+y(s-1)] / 2$, so that $T$ is a $Q$ operator over $[0,1]$. For this example,

$$
x_{1}(t)=\left\{\begin{array}{c}
0 \text { if } t \leqq 0, \\
1 / 2 \text { if } 0<t \leqq 1, \\
1 \text { if } t>1 .
\end{array} \quad x_{2}(t)=\left\{\begin{array}{c}
0 \text { if } t<0 \\
1 / 2 \text { if } 0 \leqq t<1, \\
1 \text { if } t \geqq 1 .
\end{array}\right.\right.
$$

THEOREM 4.2. Suppose that $[c, d]$ is an interval and $u_{1}$ and $u_{2}$ are functions of bounded variation such that

(i) $u_{1}(t)=u_{2}(t)=u_{1}(c)=0$ if $t<c$,

(ii) $u_{1}(t)=u_{2}(t)=u_{2}(d)$ if $t>d$, and

(iii) $u_{1}(t-)=u_{2}(t-)$ and $u_{1}(t+)=u_{2}(t+)$ for each real number $t$.

If $s$ is a real number and $g$ and $h$ are quasi-continuous functions such that $g_{L}=g$ and $h_{R}=h$, let $U g(s)$, Uh(s), and $U y(s)$, where $y=g+h$, denote the numbers $\int_{c}^{d+} g(s-t) d u_{1}(t), \int_{c-}^{d} h(s-t) d u_{2}(t)$, and $U g(s)+U h(s)$, respectively. Then $U$ is a 
$Q$ operator over $[c, d]$, and $|U| \leqq 1.5\left[V_{c}^{d+}\left(u_{1}\right)+V_{c-}^{d}\left(u_{2}\right)\right]$.

Proof. We show first that if $y$ is quasi-continuous, then there is just one function which is $U y$. Suppose that $y$ is quasi-continuous and that $g, h, p, q$ is a sequence of quasi-continuous functions such that $g_{L}=g, h_{R}=h, p_{L}=p$, $q_{R}=q, g+h=y$, and $p+q=y$. Suppose that $f=g-p$; then $f_{L}=f$. But $f=q-h$, and therefore $f_{R}=f$; so $f$ is continuous. Now if $s$ is a real number, then $\int_{-\infty}^{+\infty} f(s-t) d u_{1}(t)-\int_{-\infty}^{+\infty} f(s-t) d u_{2}(t)=\int_{-\infty}^{+\infty} f(s-t) d\left[u_{1}(t)-u_{2}(t)\right]$. Since $f$ is continuous, $u_{1}-u_{2}$ is of bounded variation, and $u_{1}-u_{2}$ is zero except for a countable subset of $[c, d]$, it follows that $\int_{-\infty}^{+\infty} f(s-t) d\left[u_{1}(t)-u_{2}(t)\right]=0$, so that $\int_{c}^{d+} f(s-t) d u_{1}(t)=\int_{c-}^{d} f(s-t) d u_{2}(t)$, or $\int_{c}^{d+} g(s-t) d u_{1}(t)-\int_{c}^{d+} p(s-t) d u_{1}(t)$ $=\int_{c-}^{d} q(s-t) d u_{2}(t)-\int_{c-}^{d} h(s-t) d u_{2}(t)$, or $U g(s)-U p(s)=U q(s)-U h(s)$. Hence $U g(s)+U h(s)=U p(s)+U q(s)$; i.e., there is just one function which is $U y$. Moreover, if $|y(s-t)| \leqq M<M_{1}$ for each number $t$ in $[c, d]$, then by Lemma 2.5 the functions $p$ and $q$ may be chosen so that $|p(s-t)| \leqq 1.5 M_{1}$ and $|q(s-t)|$ $\leqq 1.5 M_{1}$ for each number $t$ in $[c, d]$; so by Theorem 2.1 of $[1],|U y(s)|$ $\leqq 1.5 M_{1}\left[V_{c}^{d+}\left(u_{1}\right)+V_{c-}^{d}\left(u_{2}\right)\right]$. The theorem now follows from Definition 1.1 and the properties of integrals (cf. Theorem 2.1 of [1]).

REMARK 4.2. Compare Theorem 4.1 with the representation given in [2]; for an expression for the norm $|T|$, see [6].

5. $Q_{1}$ operators. In this section, we show that if $T$ is a $Q_{1}$ operator and $y$ is a quasi-continuous function, then $T y$ is an integral; and we obtain a condition sufficient for the product of two $Q_{1}$ operators to be a $Q_{1}$ operator.

Theorem 5.1. For $T$ to be a $Q_{1}$ operator over the interval $[a, b]$ it is necessary and sufficient that there is a function $x$ such that

(i) $x$ is of bounded variation, $x(t)=0$ if $t \leqq a, x(t)=x(b)$ if $t>b$, and

(ii) if $y$ is quasi-continuous and $s$ is a real number, then

$$
T y(s)=\int_{b}^{a} y(s-t) d x(t) .
$$

Proof. A. Suppose that $T$ is a $Q_{1}$ operator over $[a, b]$, and let $R, L, x_{1}$, and $x_{2}$ be defined as in $\S 4$. By Definition 1.2, $R(s+)-R(s-)=2[R(s)-L(s)]$ for each real number $s$; and by Lemma $4.1 \mathrm{a}, R(s-)=L(s-)$, so that $2 R(s)$ $-R(s+)=2 L(s)-L(s-)$, or $x_{2}(s)=x_{1}(s)$ for each real number $s$. It follows from Theorem 3.3 that $x_{1}$ is of bounded variation, from Lemma 4.1a that $x_{1}(t)=0$ if $t \leqq a$ and $x_{1}(t)=x_{1}(b)$ if $t>b$, and from Theorem 4.1 that $T y(s)$ $=\int_{a}^{b} y(s-t) d x_{1}(t)$ for each quasi-continuous function $y$ and real number $s$.

B. Suppose that $x$ is a function such that (i) and (ii) of the theorem are true. By Theorem 4.2, $T$ is a $Q$ operator over $[a, b]$. Let $L$ and $R$ be defined as in $\S 4$. Now if $s$ is a real number, then $R(s)=[x(s)+x(s+)] / 2$ and $L(s)$ $=[x(s-)+x(s)] / 2$, whence $2 R(s)-R(s+)=2 L(s)-L(s-)=x(s)$; since $R(s+)=L(s+)$ and $R(s-)=L(s-)$, it follows that $R(s+)-R(s-)$ $=2[R(s)-L(s)]$ and $L(s+)-L(s-)=2[R(s)-L(s)]$. Now if $y$ is a stepfunction and $s$ is a real number, then there are a number sequence $a_{1}$, $a_{2}, \cdots, a_{2 n}$ and a real-number sequence $t_{1}, t_{2}, \cdots, t_{n}$ such that 


$$
y(s-t)=\sum_{p=1}^{n}\left[a_{2 p-1} J_{R}\left(s-t_{p}-t\right)+a_{2 p} J_{L}\left(s-t_{p}-t\right)\right] \text { if } a \leqq t \leqq b ;
$$

hence $T y(s)=\sum_{p=1}^{n}\left[a_{2_{p-1}} R\left(s-t_{p}\right)+a_{2_{p}} L\left(s-t_{p}\right)\right]$, so that $T y(s+)-T y(s-)$ $=2\left[T y_{R}(s)-T y_{L}(s)\right]$. It now follows from Lemmas $2.1,3.3 \mathrm{c}$, and 2.3 that if $y$ is quasi-continuous and $s$ is a real number, then $T y(s+)-T y(s-)$ $=2\left[T y_{R}(s)-T y_{L}(s)\right]$. Hence $T$ is a $Q_{1}$ operator over $[a, b]$. This completes the proof.

REMARK 5.1. If $T$ is a $Q_{1}$ operator over an interval $[a, b]$ then there is just one function $x$ such that

(i) $x(a)=0$ and

(ii) if $y$ is quasi-continuous and $s$ is a real number, then $T y(s)=\int_{-\infty}^{+\infty} y(s-t)$ $d x(t)$. Moreover, $x$ is of bounded variation.

THEOREM 5.2. Suppose that $U$ is a $Q_{1}$ operator over the interval $[a, b], V$ is a $Q_{1}$ operator over the interval $[c, d]$, and $T y=U(V y)$ for each quasi-continuous function $y$. Let $u$ and $v$ denote the functions such that $u(a)=v(c)=0$ and $U y(s)$ $=\int_{-\infty}^{+\infty} y(s-t) d u(t)$ and $V y(s)=\int_{-\infty}^{+\infty} y(s-t) d v(t)$ if $y$ is quasi-continuous and $s$ is a real number. If $u_{L}=u_{R}$ or $v_{L}=v_{R}$, then $T$ is a $Q_{1}$ operator over $[a+c, b+d]$.

Proof. By Corollary 3.3a, $T$ is a $Q$ operator over $[a+c, b+d]$. Suppose that $u_{L}=u_{R}$ or $v_{L}=v_{R}$. Let $R$ and $L$ denote the functions $T J_{R}$ and $T J_{L}$, respectively. Now $V J_{L}=\left(v_{L}+v\right) / 2$ and $V J_{R}=\left(v+v_{R}\right) / 2$; hence if $s$ is a real number, then $2 L(s)=\int_{a}^{b}\left[v_{L}(s-t)+v(s-t)\right] d u(t)$ and $2 R(s)=\int_{a}^{b}[v(s-t)$ $\left.+v_{R}(s-t)\right] d u(t)$, so that $2[R(s)-L(s)]=\int_{a}^{b}\left[v_{R}(s-t)-v_{L}(s-t)\right] d u(t)$. Since $v$ is of bounded variation, so is $v_{R}-v_{L}$; and if $v_{R} \neq v_{L}$, then there is a countable real-number set $K$ such that $v_{R}(t) \neq v_{L}(t)$ if and only if $t$ is in $K$. Since $u$ is of bounded variation, it follows from Theorem 3.1 of [1] and Lemma 4.2a of [1] that

$$
\begin{aligned}
4[R(s)-L(s)]= & \sum_{(t)}\left[v_{R}(s-t)-v_{L}(s-t)\right][u(t)-u(t-)] \\
& +\sum_{(t)}\left[v_{R}(s-t)-v_{L}(s-t)\right][u(t+)-u(t)] \\
= & \sum_{(t)}\left[v_{R}(s-t)-v_{L}(s-t)\right]\left[u_{R}(t)-u_{L}(t)\right] .
\end{aligned}
$$

But by hypothesis, $v_{R}=v_{L}$ or $u_{R}=u_{L}$; hence $R=L$.

Now $2 L=U\left(v_{L}+v\right)$, where $U$ is a $Q_{1}$ operator; hence if $s$ is a real number, then $2[L(s+)-L(s-)]=4\left[U v_{R}(s)-U v_{L}(s)\right]$. But $U v_{R}-U v_{L}=U\left(v+v_{R}\right)$ $-U\left(v_{L}+v\right)=2(R-L)=0$. Hence if $s$ is a real number then $L(s+)-L(s-)$ $=0$; and by Lemma 4.1a, $R(s+)=L(s+)$ and $R(s-)=L(s-)$; hence $R(s+)$ $-R(s-)=2[R(s)-L(s)]=0$. By the argument in part $\mathrm{B}$ of the proof of Theorem 5.1, it follows that $T$ is a $Q_{1}$ operator over $[a+c, b+d]$. This completes the proof. 
6. $Q_{1}$ operators having specified properties. In this section we suppose that $T$ is a $Q_{1}$ operator over an interval $[a, b]$, and that $x$ is the function of bounded variation such that $x(t)=0$ if $t \leqq a, x(t)=x(b)$ if $t>b$, and $T y(s)$ $=\int_{a}^{b} y(s-t) d x(t)$ if $y$ is quasi-continuous and $s$ is a real number. We shall obtain conditions on $x$ which are sufficient to assure that $T$ has various ones of the properties described in the following definition.

Definition 6.1. (i) The statement that $T$ is symmetric means that if $y$ is quasi-continuous and $z(t)=y(-t)$ for each real number $t$, then $T z(s)$ $=T y(-s)$ for each real number $s$.

(ii) The statement that $T$ has property $\mathrm{B}$ means that if $y$ is quasi-continuous, then $T y$ is of bounded variation.

(iii) The statement that $T$ has property $\mathrm{C}$ means that if $y$ is quasicontinuous, then $T y$ is continuous.

(iv) The statement that $T$ has property $\mathrm{D}$ means that (a) if $y$ is quasicontinuous, then $T y$ has a derivative, and (b) there is a positive number $N$ such that if $y$ is quasi-continuous, $s$ is a real number, and $M \geqq|y(s-t)|$ for each number $t$ in $[a, b]$, and $g=T y$, then $\left|g^{\prime}(s)\right| \leqq M N$.

(v) If $K$ is a set of quasi-continuous functions, then the statement that the members of $K$ are invariant under $T$ means that if $y$ is in $K$ then $T y=y$.

THEOREM 6.1. For $T$ to be symmetric, it is necessary and sufficient that $x(-t)-x(0)=x(0)-x(t)$ for each real number $t$.

Proof. A. Suppose that $T$ is symmetric. If $y$ is quasi-continuous, and $z(t)$ $=y(-t)$ for each real number $t$, then $T z(0)=T y(0)$, or $\int_{-\infty}^{+\infty} z(-t) d x(t)$ $=\int_{-\infty}^{+\infty} y(-t) d x(t)$, or $\int_{-\infty}^{+\infty}[y(t)-y(-t)] d x(t)=0$. Hence if $f$ is an odd quasicontinuous function, then $\int_{-\infty}^{+\infty} f(t) d x(t)=0$. Suppose that if $p>0$ then

$$
f_{p}(t)=\left\{\begin{aligned}
0 & \text { if } t<-p, \text { or } t=0 \text { or } t>p, \\
-1 & \text { if }-p \leqq t<0, \\
1 & \text { if } 0<t \leqq p .
\end{aligned}\right.
$$

Since $f_{p}$ is an odd function, it follows that $\int_{-\infty}^{+\infty} f_{p}(t) d x(t)=0$. Integration by parts gives the equation $\int_{-\infty}^{+\infty} x(t) d f_{p}(t)=0$. By Theorem 3.1 of [1],

$$
\begin{aligned}
{[x(-p-)-x(0)]+[x(-p)-x(0)] } & -[x(0-)-x(0)]-[x(0+)-x(0)] \\
& +[x(p)-x(0)]+[x(p+)-x(0)]=0
\end{aligned}
$$

for each positive number $p$. Consequently, upon considering a sequence of positive numbers $p_{1}, p_{2}, p_{3}, \cdots$ which converges to zero, we conclude that $x(0-)-x(0)=x(0)-x(0+)$. By a similar argument, if $p>0$, then $x(-p-)$ $-x(0)=x(0)-x(p+)$; hence $x(-p)-x(0)=x(0)-x(p)$.

B. Suppose that $x(-t)-x(0)=x(0)-x(t)$ for each real number $t$. Suppose that $y$ is quasi-continuous, $z(t)=y(-t)$ for each real number $t$, and $s$ is a real number. Then 


$$
\begin{aligned}
T z(s) & =\int_{-\infty}^{+\infty} z(s-t) d x(t)=\int_{+\infty}^{-\infty} z(s+t) d x(-t) \\
& =\int_{+\infty}^{-\infty} y(-s-t) d[x(-t)-x(0)] \\
& =-\int_{+\infty}^{-\infty} y(-s-t) d[x(t)-x(0)]=\int_{-\infty}^{+\infty} y(-s-t) d x(t)=T y(-s) .
\end{aligned}
$$

This completes the proof.

THEOREM 6.2. Suppose that if $[c, d]$ is an interval then there is a positive number $N$ such that if $s_{0}, s_{1}, \cdots, s_{m}$ is a subdivision of $[c, d]$ and $t_{0}, t_{1}, \cdots, t_{n}$ is a subdivision of $[a-d, b-c]$, then

$$
\sum_{i=0}^{m-1} \sum_{j=0}^{n-1}\left|x\left(s_{i+1}+t_{j+1}\right)-x\left(s_{i+1}+t_{j}\right)-x\left(s_{i}+t_{j+1}\right)+x\left(s_{i}+t_{j}\right)\right| \leqq N .
$$

Then $T$ has property B.

Proof. Suppose that $y$ is quasi-continuous, $[c, d]$ is an interval, and $M \geqq|y(s-t)|$ if $s$ is in $[c, d]$ and $t$ is in $[a, b]$. Let $s_{0}, s_{1}, \cdots, s_{m}$ denote a subdivision of $[c, d]$, and suppose that $\epsilon>0$. Now if $s$ is a real number, then $T y(s)=\int_{-\infty}^{+\infty} y(s-t) d x(t)=\int_{-\infty}^{+\infty} y(-t) d x(s+t)$; hence if $I$ denotes the sum $\sum_{i=0}^{m-1}\left|T y\left(s_{i+1}\right)-T y\left(s_{i}\right)\right|$, then $I=\sum_{i=0}^{m-1}\left|\int_{-\infty}^{+\infty} y(-t) d\left[x\left(s_{i+1}+t\right)-x\left(s_{i}+t\right)\right]\right|$. Let $t_{0}, t_{1}, \cdots, t_{n}$ denote a subdivision of $[a-d, b-c]$ such that if $i$ is one of the integers $0,1, \cdots, m-1$, then

$$
\begin{aligned}
\mid \int_{-\infty}^{+\infty} y(-t) d\left[x \left(s_{i+1}\right.\right. & \left.+t)-x\left(s_{i}+t\right)\right] \\
& -2^{-1} \sum_{j=0}^{n-1}\left[y\left(-t_{j}\right)+y\left(-t_{j+1}\right)\right]\left[x\left(s_{i+1}+t_{j+1}\right)-x\left(s_{i}+t_{j+1}\right)\right. \\
& \left.-x\left(s_{i+1}+t_{j}\right)+x\left(s_{i}+t_{j}\right)\right] \mid<\epsilon / m .
\end{aligned}
$$

Now $|y(-t)| \leqq M$ if $a-d \leqq t \leqq b-c$; hence $I<M N+\epsilon$, and consequently $V_{c}^{d}(T y) \leqq M N$. This completes the proof.

COROllary 6.2a. If $x$ has a derivative which is of bounded variation, then $T$ has property $\mathrm{B}$.

Proof. Suppose that $[c, d]$ is an interval. If $D$ is a subdivision $s_{0}, s_{1}, \cdots, s_{m}$ of $[c, d]$ and $E$ is a subdivision $t_{0}, t_{1}, \cdots, t_{n}$ of $[a-d, b-c]$, let $S(D, E)$ denote the sum $\sum_{i=0}^{m-1} \sum_{j=0}^{n-1}\left|x\left(s_{i+1}+t_{j+1}\right)-x\left(s_{i}+t_{j+1}\right)-x\left(s_{i+1}+t_{j}\right)+x\left(s_{i}+t_{j}\right)\right|$. Suppose that $D$ and $E$ are such subdivisions and that $\epsilon>0$. By hypothesis, $x^{\prime}$ is of bounded variation and (by Lemma 2.7) is therefore continuous; moreover, $x^{\prime}(t)=0$ if $t<a$ or $t>b$. Hence there is a positive number $\delta$ such that if 
$p$ and $q$ are real numbers and $|p-q|<\delta$, then $\left|x^{\prime}(p)-x^{\prime}(q)\right|<\epsilon$. Let $u_{0}$, $u_{1}, \cdots, u_{r}$ denote a refinement $E^{\prime}$ of $E$ such that $u_{j+1}-u_{j}<\delta, j=0,1, \cdots$, $r-1$. Now

$$
\begin{aligned}
S\left(D, E^{\prime}\right)=\sum_{i=0}^{m-1} \sum_{j=0}^{r-1} \mid \frac{x\left(s_{i+1}+u_{j+1}\right)-x\left(s_{i+1}+u_{j}\right)}{u_{j+1}-u_{j}} \\
\quad-\frac{x\left(s_{i}+u_{j+1}\right)-x\left(s_{i}+u_{j}\right)}{u_{j+1}-u_{j}} \mid\left(u_{j+1}-u_{j}\right) ;
\end{aligned}
$$

and by Lemma 2.6 ,

$$
\begin{aligned}
S\left(D, E^{\prime}\right)< & 2[(b-c)-(a-d)] \epsilon(2)^{1 / 2} \\
& +\sum_{i=0}^{m-1} \sum_{j=0}^{r-1}\left|x^{\prime}\left(s_{i+1}+u_{j}\right)-x^{\prime}\left(s_{i}+u_{j}\right)\right|\left(u_{j+1}-u_{j}\right) \\
< & 2[(b-c)-(a-d)] \epsilon(2)^{1 / 2}+\sum_{j=0}^{r-1}\left(u_{j+1}-u_{j}\right) V_{-\infty}^{+\infty}\left(x^{\prime}\right) \\
< & {[(b-c)-(a-d)]\left[2 \epsilon(2)^{1 / 2}+V_{-\infty}^{+\infty}\left(x^{\prime}\right)\right] . }
\end{aligned}
$$

But since $E^{\prime}$ is a refinement of $E$, it follows that $S(D, E) \leqq S\left(D, E^{\prime}\right)$. Consequently, $S(D, E) \leqq[(b-c)-(a-d)] V_{-\infty}^{+\infty}\left(x^{\prime}\right)$. By Theorem 6.2, $T$ has property B. This completes the proof.

TheOREM 6.3. For $T$ to have property $\mathrm{C}$, it is necessary and sufficient that $x$ is continuous.

Proof. Suppose that $T$ has property $\mathrm{C}$; then $T J_{L}$ is continuous and $T J_{R}$ is continuous. Since $T J_{L}=\left(x_{L}+x\right) / 2$ and $T J_{R}=\left(x+x_{R}\right) / 2$, it follows that $x$ is continuous. Suppose now that $x$ is continuous and that $\epsilon>0$. Since $x(t)=0$ if $t \leqq a$ and $x(t)=x(b)$ if $t>b$, there is a positive number $\delta$ such that if $p$ and $q$ are real numbers and $|p-q|<\delta$, then $|x(p)-x(q)|<\epsilon$. Now if $s_{1}$ and $s_{2}$ are two real numbers and $\left|s_{1}-s_{2}\right|<\delta$, and $g(t)=x\left(s_{1}+t\right)-x\left(s_{2}+t\right)$ for each real number $t$, then $|g(t)|<\epsilon$ for each real number $t$, and $V_{-\infty}^{+\infty}(g) \leqq 2 V_{a}^{b}(x)$. Suppose that $y$ is quasi-continuous, $s_{1}$ is a real number, and $[c, d]$ is an interval containing $s_{1}$. If $s_{2}$ is in $[c, d]$, then $\left|T y\left(s_{1}\right)-T y\left(s_{2}\right)\right|=\mid \int_{a-d}^{b-c} y(-t) d\left[x\left(s_{1}+t\right)\right.$ $\left.-x\left(s_{2}+t\right)\right] \mid$; so by Lemma $4.2 \mathrm{a}$ of [1], $T y\left(s_{2}\right) \rightarrow T y\left(s_{1}\right)$ as $s_{2} \rightarrow s_{1}$. That is to say, $T y$ is continuous, or $T$ has property $\mathrm{C}$. This completes the proof.

THEOREM 6.4. For $T$ to have property $\mathrm{D}$, it is necessary and sufficient that $x$ has a derivative which is of bounded variation. Moreover, if $T$ has property $\mathrm{D}$, $y$ is quasi-continuous, and $g=T y$, then $g^{\prime}$ is continuous, and $g^{\prime}(s)=\int_{a}^{b} y(s-t)$ $d x^{\prime}(t)$ for each real number $s$.

Proof. A. Suppose that $T$ has property D. If $y$ is quasi-continuous and $g=T y$, let $U y$ denote the function $g^{\prime}$. It follows from Definition 1.1 and (iv) of 
Definition 6.1 that $U$ is a $Q$ operator over $[a, b]$. By Theorem 3.3, if $y$ is quasicontinuous, then $U y$ is quasi-continuous; and by Lemma 2.7, $U y$ is continuous; i.e., if $y$ is quasi-continuous and $g=T y$, then $g^{\prime}$ is continuous. In particular, $U J_{R}$ and $U J_{L}$ are continuous; so by Lemma 4.1a, $U J_{R}=U J_{L}$, and it follows that $U$ is a $Q_{1}$ operator over $[a, b]$. Let $u$ denote the function $U J$. If $y$ is quasi-continuous and $s$ is a real number, then $U y(s)=\int_{a}^{b} y(s-t) d u(t)$, But if $R=T J_{R}$, then $u=R^{\prime}$; and since $R$ has a derivative, $R$ is continuous so that $x=R$, whence $x^{\prime}=R^{\prime}=u$. Consequently, if $y$ is quasi-continuous and $s$ is a real number, then $U y(s)=\int_{a}^{b} y(s-t) d x^{\prime}(t)$. Moreover, since $U$ is a $Q_{1}$ operator, $x^{\prime}$ is of bounded variation.

B. Suppose that $x$ has a derivative which is of bounded variation. By Lemma $2.7, x^{\prime}$ is continuous; and we observe that $x^{\prime}(t)=0$ if $t \leqq a$ or $t \geqq b$. Suppose that $\epsilon>0$, and let $h$ denote a real number other than zero such that if $p<q$ and $q-p \leqq|h|$ then $\left|x^{\prime}(p)-x^{\prime}(q)\right|<\epsilon$. For each real number $t$, let $g_{h}(t)$ denote the number $[x(t+h)-x(t)] / h$. By Lemma 2.6, $\left|g_{h}(t)-x^{\prime}(t)\right|<2 \epsilon$ for each real number $t$; and by Lemma 2.9 , the total variation of $g_{h}$ is less than or equal to the total variation of $x^{\prime}$. Now if $y$ is quasi-continuous and $s$ is a real number, then $[T y(s+h)-T y(s)] / h=\int_{-\infty}^{+\infty} y(-t) d g_{h}(s+t)$; and by Lemma 4.2a of [1], $[T y(s+h)-T y(s)] / h \rightarrow \int_{-\infty}^{+\infty} y(-t) d x^{\prime}(s+t)=\int_{a}^{b} y(s-t) d x^{\prime}(t)$ as $h \rightarrow 0$. If $y$ is quasi-continuous and $s$ is a real number, let $T^{\prime} y(s)$ denote the number $\int_{a}^{b} y(s-t) d x^{\prime}(t)$. Then $T^{\prime}$ is a $Q_{1}$ operator over $[a, b]$, such that if $y$ is quasi-continuous and $g=T y$, then $g^{\prime}=T^{\prime} y$. Hence $T$ has property $\mathrm{D}$. This completes the proof.

Theorem 6.5. Suppose that $n$ is a positive integer. For all polynomials of degree $n$ or less to be invariant under $T$, it is necessary and sufficient that $x(b)=1$ and $\int_{a}^{b} t^{p} d x(t)=0$ for $p=1,2, \cdots, n$. If $T$ is symmetric, and $n$ is an even integer, and all polynomials of degree $n$ or less are invariant under $T$, then all polynomials of degree $n+1$ or less are invariant under $T$.

Proof is omitted, since the theorem readily follows if for each polynomial $y$ and real number $s$ we consider the Maclaurin expansion of $y(s-t)$ in powers of $t$.

7. A family of smoothing operators and differentiating operators. In this section we suppose that $n$ is a positive integer and $[a, b]$ is an interval of unit length. We shall exhibit a $Q_{1}$ operator $T$ on $[a, b]$ such that

(i) all polynomials of degree 2 or less are invariant under $T$, and

(ii) if $y$ is quasi-continuous, then $T y$ has an $n$th derivative.

Let polynomials $u, v$, and $w$ be defined as follows. If $t$ is a real number, then

$$
\begin{aligned}
2 u(t) & =1+[2 t-(a+b)] \sum_{p=0}^{n}\left(\begin{array}{c}
2 p \\
p
\end{array}\right)(t-a)^{p}(b-t)^{p}, \\
v(t) & =(n+2)\left(\begin{array}{c}
2 n+3 \\
n+1
\end{array}\right)(t-a)^{n+1}(b-t)^{n+1},
\end{aligned}
$$


and

$$
w(t)=(n+2)\left(\begin{array}{c}
2 n+3 \\
n+1
\end{array}\right)(t-a)^{n+2}(b-t)^{n+1} .
$$

Let $A=(2 n+5)[2 a b+(n+1) /(2 n+3)]$ and $B=(a+b+A) / 2$. Let $x$ denote the function such that $x(t)=0$ if $t<a, x(t)=1$ if $t>b$, and $x(t)=u(t)+B v(t)$ $-A w(t)$ if $a \leqq t \leqq b$. If $y$ is quasi-continuous and $s$ is a real number, let $T y(s)$ denote the number $\int_{a}^{b} y(s-t) d x(t)$, and let $T^{\prime} y(s)=\int_{a}^{b} y(s-t) d x^{\prime}(t)$.

By some rather tedious manipulation, it can be seen that $x$ has a continuous $n$th derivative which is of bounded variation, and that $x(a)=0, x(b)=1$, $\int_{a}^{b} t d x(t)=0$, and $\int_{a}^{b} t^{2} d x(t)=0$, so that polynomials of degree 2 or less are invariant under $T$. Moreover, if $a=-1 / 2$, then $T$ is symmetric, so that polynomials of degree 3 or less are invariant under $T$. If $y$ is quasi-continuous and $g=T y$, then $g^{\prime}=T^{\prime} y$.

In particular, if $n=3$ and $a \leqq t \leqq b$, then

$$
\begin{aligned}
x(t)= & 0.5+0.5(2 t-a-b)\left[1+2(t-a)(b-t)+6(t-a)^{2}(b-t)^{2}\right. \\
& \left.+20(t-a)^{3}(b-t)^{3}-140(22+99 a b)(t-a)^{4}(b-t)^{4}\right] \\
& +315(a+b)(t-a)^{4}(b-t)^{4},
\end{aligned}
$$

and

$$
\begin{aligned}
x^{\prime}(t)=140(t-a)^{3}(b-t)^{3}[(45+198 a b)+ & 9(a+b)(a+b-2 t) \\
& -(198+891 a b)(t-a)(b-t)] .
\end{aligned}
$$

In particular, if $n=3, a=-1 / 2$, and $-1 / 2 \leqq t \leqq 1 / 2$, then

$$
x(t)=0.5+t\left[1+2\left(.25-t^{2}\right)+6\left(.25-t^{2}\right)^{2}+20\left(.25-t^{2}\right)^{3}\right.
$$

and

$$
\left.+385\left(.25-t^{2}\right)^{4}\right]
$$

$$
x^{\prime}(t)=315\left(.25-t^{2}\right)^{3}\left[11\left(.25-t^{2}\right)-2\right],
$$

and $T$ is a limit of the "most powerful" smoothing operators (i.e., operators with minimum smoothing coefficients) described, e.g., in [4]. For this instance the operators $T$ and $T^{\prime}$ have been used with quite satisfactory results on a digital computer with experimental data, the integrals being approximated by an approximating sum as in [1], with the subdivision $-.50,-.45,-.40$, $\cdots, .45, .50$ of the interval $[-1 / 2,1 / 2]$.

If $n=3, a=0$, and $0 \leqq t \leqq 1$, then

$$
\begin{aligned}
x(t)=0.5+0.5(2 t-1)\left[1+2 t(1-t)+6 t^{2}(1-t)^{2}+20 t^{3}(1-t)^{3}\right. \\
\\
\left.-3080 t^{4}(1-t)^{4}\right]+315 t^{4}(1-t)^{4},
\end{aligned}
$$

and 


$$
x^{\prime}(t)=2520 t^{3}(1-t)^{3}\left(3-12 t+11 t^{2}\right) ;
$$

in this instance $T$ is not symmetric, and the application of the operators $T$ and $T^{\prime}$ to experimental data does not give results as satisfactory as those obtained with the symmetric operator previously described.

\section{REFERENCES}

1. R. E. Lane, The integral of a function with respect to a function, Proc. Amer. Math. Soc. vol. 5 (1954) pp. 59-66.

2. H. S. Kaltenborn, Linear functional operations on functions having discontinuities of the first kind, Bull. Amer. Math. Soc. vol. 40 (1934) pp. 702-708.

3. T. H. Hildebrandt, On bounded linear functional operations, Trans. Amer. Math. Soc. vol. 36 (1934) pp. 868-875.

4. Morton D. Miller, Elements of graduation, Actuarial Society of America, 1946.

5. N. Bourbaki, Éléments de mathématique; IX, Première Partie, Les structures fondamentales de l'analyse; Livre IV, Fonctions d'une variable réelle; Hermann et Cie., Paris, 1949, Chapter 2, pp. 59-77.

6. G. Fichtenholz, Sur une généralisation de l'intégrale de Stieltjes, Doklady, Akad. Nauk SSSR vol. 3 (12) (1936) pp. 95-100.

The University of Texas, Austin, Texas 